\title{
Cooling balloons with liquid nitrogen
}

\author{
A. J. Moreno, H. Ferrari, and V. Bekeris \\ Departamento de Física, Laboratorio de Bajas Temperaturas, Facultad de Ciencias Exactas y Naturales, \\ Universidad de Buenos Aires, Pabellón I, Ciudad Universitaria, C1428EGA Buenos Aires, Argentina \\ and CONICET, Av. Rivadavia 1917, C1033AAJ, Ciudad de Buenos Aires, Argentina
}

(Received 5 March 2010; accepted 9 July 2010)

\begin{abstract}
We present an undergraduate level experiment in which the radius of a rubber balloon is measured as it is cooled with liquid nitrogen. For balloons filled with simple gases that condense at liquid nitrogen temperatures, we found that the volume decreases linearly with time. We compared our measurements with a simplified model based on elementary kinetic theory and thermodynamics that explains this behavior. Students are encouraged to test the validity of the model by repeating the experiment using gas mixtures and gases that do not condense at liquid nitrogen temperatures. (C) 2010 American Association of Physics Teachers.

[DOI: 10.1119/1.3473787]
\end{abstract}

\section{INTRODUCTION}

There are many qualitative experiments that involve the use of liquid nitrogen to cool objects. ${ }^{1-3}$ In some of these experiments, rubber objects, and even fruit or flowers, are cooled to show their mechanical properties at very low temperatures. In other experiments, the magnetic properties of oxygen in liquid nitrogen are shown qualitatively. ${ }^{4}$

A typical and appealing demonstration consists of cooling a rubber balloon filled with gas using liquid nitrogen to show the reduction of the balloon's volume as the gas is cooled and its increase as the balloon is left to return to room temperature. ${ }^{1-3}$ In these demonstrations, the change of volume is presented but no quantitative determinations are made because the dynamics of rubber is very complex. It is known that rubber does not follow Hooke's law and that stretched rubber exerts a force that is nonconservative, resulting in a hysteresis loop in force versus length measurements. ${ }^{5}$ Odd effects are found in experiments where two rubber balloons, inflated to different volumes, are connected through their nozzles. The air flows either from the larger (fuller) balloon to the smaller (emptier) balloon, or from the smaller to the larger balloon, showing that the relation between the internal pressure and the volume of a rubber balloon is complex. ${ }^{6-8}$

In this paper, we present a quantitative procedure in which the volume of a balloon, filled with a known gas, is measured as a function of time as it is cooled when put in contact with liquid nitrogen. We also present a simple model that describes the measured dependence of the cube of the radius of the balloon with time. The model assumes that the gases condense at liquid nitrogen temperature $T_{\mathrm{LN}}$. The behavior of balloons filled with mixtures or with gases that do not condense at $T_{\mathrm{LN}}$ is also discussed to show the limits of the model.

\section{EXPERIMENTAL SETUP}

The purpose of this experiment is to measure the variation of the volume of a balloon as it is cooled while in contact with liquid nitrogen. A movie or a sequence of photos of the balloon can be taken during this process using a digital camera. The radius of the balloon as a function of time can be determined from these images with image processing software or a simple MATLAB program, as we will explain.

A schematic of the experimental setup is shown in Fig. 1. We used common rubber or latex balloons filled with indus- trially pure gases (oxygen, argon, propane, nitrogen, and helium) and air, with typical initial volumes of $2 \times 10^{-3} \mathrm{~m}^{3}$. A rectangular expanded polypropylene container is used to contain the liquid nitrogen. The balloon was placed over the liquid nitrogen and a pin was used to fix the balloon's nozzle to the wall of the polystyrene container to prevent it from moving and rotating while it is floating over the nitrogen. A digital camera fixed to a tripod was used to film the balloon or to take photos at fixed periods of time. The camera was focused on the balloon along its axis containing the nozzle. In this way, we can assume that the shape of the balloon, as is viewed by the camera, is circular. It is convenient not to over inflate the balloon so that its shape remains approximately spherical.

To measure the radius of the balloon, we processed the movies using a MATLAB program that converts each image of the movie into a data matrix and uses an algorithm to find the contour of the balloon. ${ }^{9}$ A circle was fitted to this contour to obtain the radius of the balloon in each image. By knowing the number of images the camera takes per second (typically 15), we can obtain the variation of the radius with time. A simpler alternative, which is more time consuming, is to process the photos with an image processing program (such as IMAGE J or PHOTOSHOP) and to measure the radius of the balloon using one of the tools provided by the software.

Caution should be taken when handling liquid nitrogen, which can cause frostbite. It is mandatory to wear appropriate gloves and eye protection. It is advisable to do the experiment in a well ventilated room because as liquid nitrogen evaporates, it can displace air, causing suffocation. We also used propane gas, which is highly flammable, and therefore extreme caution should be taken when handling it. It is advisable to avoid open flames, electrical sparks, or any high temperature source that could ignite the gas.

\section{MODEL}

Although the physics of the mechanical properties of rubber is complex, we presented the following simple model. We considered $N_{0}$ molecules of an ideal gas inside a balloon at room temperature $T_{\mathrm{amb}}$, atmospheric pressure $P_{\mathrm{atm}}$, and volume $V$. The temperature gradient established as the balloon is in contact with the cryogenic liquid will be approximated as an average temperature $T$ inside the balloon. There is a small change in the internal pressure of the balloon due 

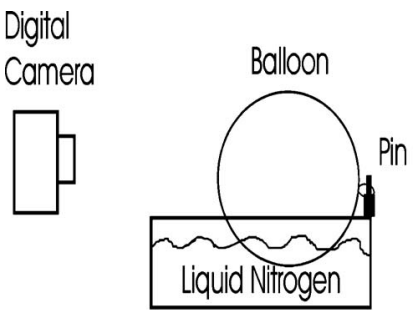

Expanded polvestyrene tray

Fig. 1. Experimental setup.

to cooling, but we have measured this change with a manometer and found it to be negligible [(from $\left(P_{\mathrm{atm}}+0.01 P_{\mathrm{atm}}\right)$ to $\left.\left(P_{\mathrm{atm}}+0.005 P_{\mathrm{atm}}\right)\right]$, and we will assume that the pressure inside the balloon is $\cong P_{\text {atm }}$.

We assumed a quasistationary regime in which a fraction $\delta$ of gas molecules that collide with the internal wall of the balloon (in contact with the liquid nitrogen) condense and are no longer part of the gas. ${ }^{10}$ This fraction, to be determined in the experiment, is the net condensation rate, which is related to the difference in fluxes of molecules moving toward or outward from the liquid (condensed gas) surface. The number $N_{g}$ of molecules of the gas in the balloon changes in time as a function of the flux of molecules arriving and condensing at the cold surface in contact with the liquid nitrogen,

$$
N_{g}(t)=N_{0}-N_{w}(t),
$$

where $N_{w}$ is the number of molecules that collide with the cold wall and condense.

The molecular flux $\Phi$ is the number of molecules that arrive at a surface per unit time per unit area and is equal to ${ }^{11}$

$$
\Phi=\frac{1}{4}\langle v\rangle \frac{N}{V},
$$

where $\langle v\rangle$ is the average speed of the molecules at temperature $T$. If we assume that the molecules collide with a constant area $A$, we can obtain how the number of molecules varies with time,

$$
\frac{d N_{w}}{d t}=\frac{A}{4} \delta\langle v\rangle \frac{N_{g}}{V} .
$$

During the experiment, the surface of the balloon in contact with the liquid nitrogen changed very little, and we assumed it is constant and the shape of the balloon can be approximated by a sphere until it is almost fully deflated. From Eqs. (1) and (3), we obtained

$$
\frac{d N_{g}(t)}{d t}=\frac{d\left(N_{0}-N_{w}(t)\right)}{d t}=-\frac{d N_{w}(t)}{d t} .
$$

Thus, we obtained

$$
\frac{d N_{g}}{d t}=-\frac{d N_{w}}{d t}=-\frac{A}{4} \delta\langle v\rangle \frac{N_{g}}{V} .
$$

If we assume an ideal gas at constant temperature $T$ and atmospheric pressure, we have

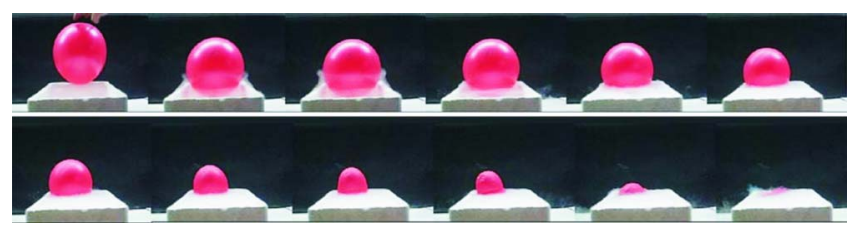

Fig. 2. Typical sequence of balloon deflation.

$$
\frac{d N_{g}}{d t}=-\frac{A}{4} \delta\langle v\rangle \frac{P_{\mathrm{atm}}}{k_{B} T}=\text { constant },
$$

where $k_{B}$ is Boltzmann's constant. Therefore, the number of molecules of the gas inside the balloon varies linearly with time as

$$
N_{g}(t)=N_{0}-\left(\frac{A}{4} \delta\langle v\rangle \frac{P_{\mathrm{atm}}}{k_{B} T}\right) t .
$$

Because the temperature and pressure are assumed to be constant, the volume of the gas $V_{g}$ is proportional to the number of molecules,

$$
V_{g}(t)=V_{0}-\left(\frac{A \delta\langle v\rangle}{4}\right) t .
$$

For a spherical balloon,

$$
V_{g}(t)=\frac{4}{3} \pi R^{3}(t)=V_{0}-\left(\frac{A \delta\langle v\rangle}{4}\right) t .
$$

Therefore, the volume of the balloon (the cube of the radius) decreases linearly with time,

$$
R^{3} \propto a-b t .
$$

\section{RESULTS AND DISCUSSION}

We first performed the experiment using different balloons filling each one with similar volumes of a single gas that condenses at $T_{\mathrm{LN}}$, that is, oxygen, argon, or propane. In Fig. 2 we show a typical sequence of photos of the cooling of a balloon. We assumed that the balloon maintains approximately spherical during the cooling process until the balloon is nearly completely deflated. The lower part of the balloon, which is in contact with liquid nitrogen, loses its elasticity because at very low temperatures rubber becomes frozen and very brittle. In Fig. 2 we consider our model to be applicable from the second photo of the upper row, when the balloon touches the liquid nitrogen, to the third photo from the left of the bottom row (ninth photo of the sequence) when the balloon loses its spherical shape.

After carefully choosing the useful range of images from each sequence, we obtained the relevant parameters. We used the measured dimensions of the container to scale pixels into length units. In Fig. 3 we show the cube of the radius of the balloon $R^{3}$ as a function of time for several gases. The initial volume $V_{0}$ of each balloon fixes the initial ordinate. It can be seen from Fig. 3 that there is an initial decrease in the radius of the balloon due to the formation of a temperature gradient inside it. A linear regime then appears (between the arrows) where the gas is condensing. We identified the final nonlinear part of the curves with the balloon almost deflated. It is very important to correlate each image with its point on the curve to clearly identify when the balloon is no longer spherical 


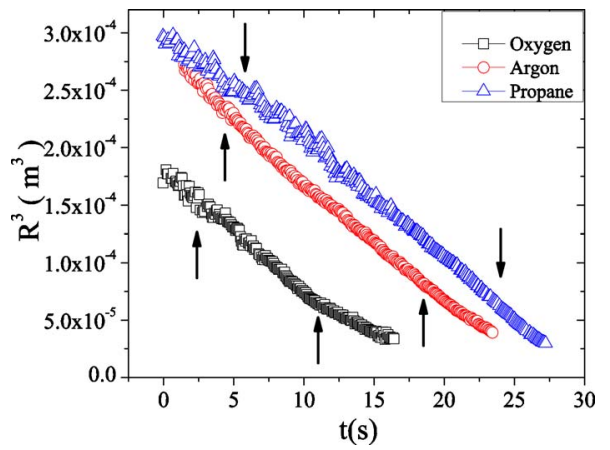

Fig. 3. Cube of the radius of the balloons versus time for balloons filled with oxygen, argon, or propane. The leftmost arrow indicates the beginning of the approximately linear variation of $R^{3}$ with time after the inner temperature is established. The rightmost arrow indicates when the balloon loses its spherical shape due to freezing of the rubber.

due to the reduction of the volume and the loss of elasticity of the rubber. We disregarded the first and last parts of the curves and focused on the approximately linear part depicted between the arrows. Experimental results agree with our model, showing a linear dependence of $R^{3}$ with time as expected from Eq. (10).

We used Eq. (9) and assumed the average temperature inside the balloon to be $100 \mathrm{~K}$ to obtain the values for the slope $b$ shown in Table I. The coefficient $\delta$ is smaller than 1 as expected. It is difficult to obtain a precise value for the area $A$ [see Eq. (9) ]. We estimated $A$ by putting a balloon in contact with liquid nitrogen for a short time and then removing it. The area that was in contact with the liquid nitrogen was cold enough to quickly condense water vapor present in air. We did a quick measurement of the diameter of the condensed spot. Also we compared our measurement with an estimate using Archimedes' principle, taking into account the weight of the deflated balloon, the weight of the gas inside it, and the buoyant force due to immersion in liquid nitrogen. We found that the fraction $\delta$ of molecules that condense after colliding with the cold area does not depend strongly on the average temperature inside the balloon. As an example, for oxygen $\delta$ varies from $2 \times 10^{-4}$ for $T=300 \mathrm{~K}$ to $4 \times 10^{-4}$ for $T=80 \mathrm{~K}$. A detailed discussion of these values requires kinetic theory and molecular dynamics simulations, ${ }^{12,13}$ which are beyond the scope of this work.

Some parts of the curves seem to be noisy, but this noise is an artifact due to the image processing. In some of the images, the mist arising from the liquid nitrogen or some buoyant movement of the balloon due to the boiling nitrogen introduced additional error in the fit of the circumference. Analyzing single photos with an image processor reduces noise, but is time consuming.

Table I. Slopes and the fraction of molecules $\delta$ that condense after colliding with the cold area.

\begin{tabular}{lcc}
\hline \hline & \multicolumn{1}{c}{$\begin{array}{c}\text { Slope } \\
\left(\mathrm{m}^{3} / \mathrm{s}\right)\end{array}$} & $\delta$ \\
\hline Oxygen & $-(1.08 \pm 0.01) \times 10^{-5}$ & $(3 \pm 1) \times 10^{-4}$ \\
Argon & $-(1.052 \pm 0.001) \times 10^{-5}$ & $(4 \pm 1) \times 10^{-4}$ \\
Propane & $-(1.029 \pm 0.001) \times 10^{-5}$ & $(4 \pm 1) \times 10^{-4}$ \\
\hline \hline
\end{tabular}

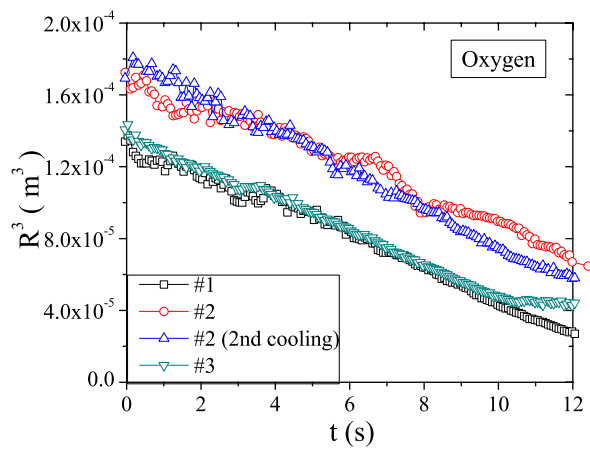

Fig. 4. $R^{3}$ versus time of balloons filled with oxygen (balloon 1, squares; balloon 2, circles; and balloon 3, down triangles) and a second cooling of balloon 2 after it was warmed up to room temperature (up triangles).

To test the reproducibility of our results, we cooled different balloons and filled them with oxygen to similar initial volumes. In Fig. 4 we show $R^{3}$ versus $t$ for different balloons and a measurement cooling the same balloon after letting it return to room temperature. The balloons show very similar behavior.

To further investigate the limitations of our model, we performed our experiment filling different balloons with either nitrogen or helium. These gases do not condense when they are put in contact with liquid nitrogen because their boiling temperature is equal to or less than that of liquid nitrogen. In Fig. 5 we show our results for balloons with a mixture of gases, that is, air. For the balloons filled with helium or nitrogen, we can observe an initial radius reduction due to cooling. After this transient behavior, the balloon maintains a constant radius. There is an initial volume $V_{0}$ of the balloon when it is at room temperature $T_{\mathrm{amb}}$ and a final volume $V_{f}$ when the temperature gradient is established. This result shows that there is no further change in the balloon's volume once the internal temperature gradient is established. Most gases in air condense so that the radius decreases with time, but $R^{3}$ does not follow a linear dependence.

Our model cannot be applied to these gases because nitrogen and helium do not condense at $T_{\mathrm{LN}}$ (and atmospheric pressure) and air is a mixture of gases. The boiling temperature of a mixture of oxygen and nitrogen depends on its composition, and it varies as the gas condenses into a liquid. ${ }^{14}$ The composition of the liquid is different from the composition of the gas in equilibrium with it. For example, if the gas has a composition of $80 \% \mathrm{~N}$ and $20 \% \mathrm{O}$ and is cooled below $79 \mathrm{~K}$, the liquid formed will have a composi-

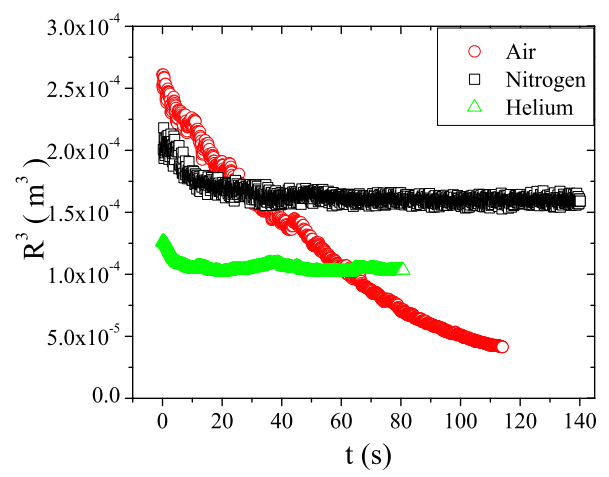

Fig. 5. $R^{3}$ versus time for balloons filled with nitrogen, helium, and air. 
tion of $53 \% \mathrm{~N}$ and $47 \% \mathrm{O}$ (see Ref. 14, p. 14.). In our model, a fixed fraction of the number of molecules that collide with the cold wall condense; for a balloon filled with air, a variable fraction of molecules (strongly dependent on the particular mixture of gases) that collide with the cold wall condense, and this time dependent fraction modifies the volume time dependence.

\section{ACKNOWLEDGMENTS}

The authors acknowledge C. Acha and G. Pasquini for valuable discussions and suggestions. This work was partially supported by UBACyT X13.

${ }^{1}$ R. G. Hunt and G. L. Salinger, "Qualitative demonstrations and experiments using liquid nitrogen,” Phys. Teach. 7 (5), 289-290 (1969).

${ }^{2}$ J. Hendricks, D. Lee, and M. Rugheimer, "Liquid nitrogen demonstrations," Am. J. Phys. 39 (7), 844-845 (1971).

${ }^{3}$ J. T. Dickinson, "Liquid nitrogen in a balloon," Phys. Teach. 15 (6), 361-362 (1977).

${ }^{4}$ R. Simmonds, K. Browning, A. Rinker, T. Gastouniotis, and D. Ion, "Demonstrating paramagnetism using liquid nitrogen," Phys. Teach. 32
(6), 374-375 (1994).

${ }^{5}$ B. Denardo and R. Masada, "Rubber hysteresis experiment," Phys. Teach. 28 (7), 489-491 (1990).

${ }^{6}$ D. R. Merritt and F. Weinhaus, "The pressure curve for a rubber balloon," Am. J. Phys. 46 (10), 976-977 (1978).

${ }^{7}$ E. Verron and G. Marckmann, "Numerical analysis of rubber balloons," Thin-Walled Struct. 41 (8), 731-746 (2003).

${ }^{8}$ Y. Levin and F. L. da Silveira, "Two rubber balloons: Phase diagram of air transfer," Phys. Rev. E 69, 051108-1-4 (2004).

${ }^{9}$ See 〈www.mathworks.com/products/image/demos.html?file=/products/ demos/shipping/images/ipexradius.html $>$.

${ }^{10} \mathrm{~T}$. Tsuruta and G. Nagayama, "A microscopic formulation of condensation coefficient and interface transport phenomena," Energy 30, 795-805 (2005).

${ }^{11}$ F. W. Sears and G. L. Salinger, Thermodynamics, Kinetic Theory and Statistical Thermodynamics, 3rd ed. (Addison-Wesley, Reading, MA, 1986).

${ }^{12}$ M. Bond and H. Struchtrup, "Mean evaporation and condensation coefficients based on energy dependent condensation probability," Phys. Rev. E 70, 061605-1-21 (2004).

${ }^{13}$ M. Matsumoto, "Molecular dynamics of fluid phase change," Fluid Phase Equilib. 144, 307-314 (1998).

${ }^{14}$ E. Wiberg, N. Wiberg, and A. Holleman, Inorganic Chemistry, 101st ed. (Academic, San Diego, 2001), p. 14.

\section{AJP SUBMISSION INFORMATION}

Authors interested in submitting a manuscript to the American Journal of Physics should first consult the following two documents:

Statement of Editorial Policy at http://www.kzoo.edu/ajp/docs/edpolicy.html

Information for Contributors at http://www.kzoo.edu/ajp/docs/information.html 